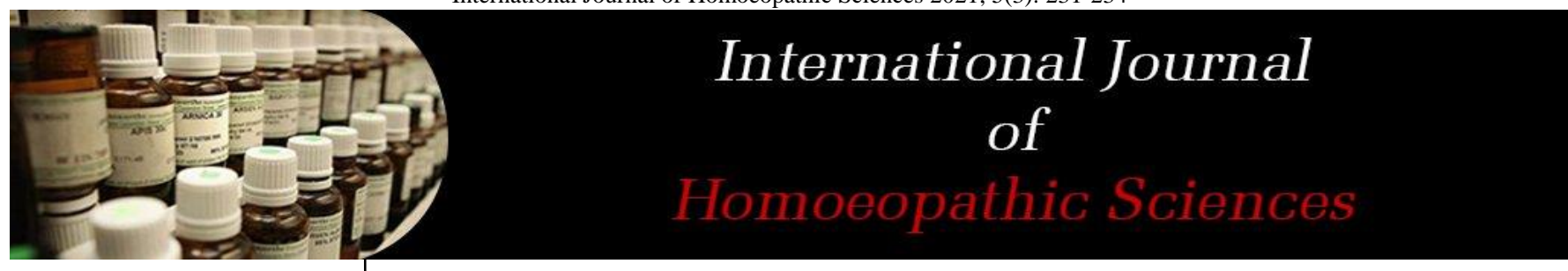

E-ISSN: $2616-4493$ P-ISSN: 2616-4485 www.homoeopathicjournal.com IJHS 2021; 5(3): 231-234

Received: 13-05-2021

Accepted: 15-06-2021

Dr. Kajal

Assistant Professor

Dept. Practice of Medicine

Government Homeopathic

Medical College and Hospital.

Muzaffarpur, Bihar, India
(P.G section), RBTS

\section{Rheumatoid arthritis: A brief overview}

\author{
Dr. Kajal \\ DOI: https://doi.org/10.33545/26164485.2021.v5.i3d.428
}

\section{Abstract}

Rheumatoid arthritis (RA) is a chronic, inflammatory, systemic autoimmune disease, affecting the joints with varying severity among patients. The risk factors include age, gender, genetics, and environmental exposure (cigarette smoking, air pollutants, and occupational). Many complications can follow, such as permanent joint damage requiring arthroplasty, rheumatoid vasculitis, and Felty syndrome requiring splenectomy if it remains unaddressed. As there is no cure for RA, the treatment goals are to reduce the pain and stop/slow further damage. Here, we present a brief summary of various past and present treatment modalities to address the complications associated with RA.

Keywords: rheumatoid arthritis, systemic autoimmune disease, rheumatoid vasculitis

\section{Introduction}

Rheumatoid arthritis (RA) is a chronic, symmetrical, inflammatory autoimmune disease that initially affects small joints, progressing to larger joints, and eventually the skin, eyes, heart, kidneys, and lungs. Often, the bone and cartilage of joints are destroyed, and tendons and ligaments weaken ${ }^{[1]}$. All this damage to the joints causes deformities and bone erosion, usually very painful for a patient. Common symptoms of RA include morning stiffness of the affected joints for $>30 \mathrm{~min}$, fatigue, fever, weight loss, joints that are tender, swollen and warm, and rheumatoid nodules under the skin. The onset of this disease is usually from the age of 35 to 60 years, with remission and exacerbation. It can also afflict young children even before the age of 16 years, referred to as juvenile RA (JRA), which is similar to RA except that rheumatoid factor is not found ${ }^{[2-5]}$. In the West, the prevalence of RA is believed to be $1-2 \%{ }^{[5,6]}$ and $1 \%$ worldwide ${ }^{[7]}$. Clinically, the diagnosis of RA can be differentiated from osteoarthritis (OA) as the affected areas in RA are the proximal interphalangeal (PIP) and metacarpophalangeal (MP) joints; OA typically affects the distal interphalangeal (DIP) joint (Fig. 1). OA is the most common type of arthritis and is caused by wear and tear rather than an autoimmune condition. It has no effects on the lungs, heart, or immune system. In addition, OA typically affects only one side of the body, as opposed to the symmetrical nature of RA. Another differentiating factor is that RA patients suffer from persistent morning stiffness for at least $\geq 1 \mathrm{~h}$. Patients with OA may have morning stiffness, but this typically resolves or decreases within $20-30 \mathrm{~min}{ }^{[8,9]}$. The goals of treatment for RA are to reduce joint inflammation and pain, maximize joint function, and prevent joint destruction and deformity. Treatment regimens consist of combinations of pharmaceuticals, weight bearing exercise, educating patients about the disease, and rest. Treatments are generally customized to a patient's needs and depend on their overall health. This includes

factors such as disease progression, the joints involved, age, overall health, occupation, compliance, and education about the disease ${ }^{[10]}$. This review briefly highlights the classic and current treatment options available to address the discomfort/complications of RA. An exhaustive review was recently published by Smolen et al. ${ }^{[11]}$.

Homeopathy shows remarkable results in mild to moderate cases of rheumatoid arthritis. However, in severe cases of rheumatoid arthritis with advanced deformities, Homeopathy helps manage the symptoms. Homeopathic medicines for rheumatoid arthritis help manage pain and stiffness in joints, but the deformities cannot be reversed.

Dr. Kajal

Assistant Professor

Dept. Practice of Medicine

(P.G section), RBTS

Government Homeopathic

Medical College and Hospital.

Muzaffarpur, Bihar, India

\section{What are its symptoms?}

Joint symptoms: The main symptoms are pain in joints, swelling in joints, stiffness of joints which mostly appear in morning and after periods of inactivity. In this, multiple joints are involved in a symmetrical manner i.e. involving the same joints on both sides of the body. 
The symptoms initially start from small joints of hands or feet and may progress to other joints like wrist, elbow, shoulder, knee, hip and ankle.

The Affected joint is also red, warm and tender to touch in flare up phase. Loss of joint function, mobility and deformity appear in later stages if not treated well in time. Few general signs and symptoms that may attend joint involvement include weakness, loss of appetite, weight loss, fever, muscle aches, depression and anaemia.

\section{Diagnostic criteria}

The diagnostic criteria for rheumatoid arthritis is as follows: Any four among the following must be present to diagnose rheumatoid arthritis:

1. Arthritis of multiple joint (3-4 different joints or more than that) for a period of more than 6 weeks

2. Morning stiffness that last longer than 30 minutes

3. Symmetrical involvement of the same joints on both sides of the body

4. Arthritis of wrists, hands or finger joints

5. Development of rheumatoid nodules under the skin

6. Positive serum rheumatoid factor

7. Radiographic changes suggesting joint erosion

\section{Joint deformities}

The main joint deformities that can develop in rheumatoid arthritis are as follows:

1. Swan neck deformity: In this deformity the joint in finger closest to fingertip gets bent towards the palm (flexion of distal inter-phalangeal joint) and the joint in finger nearest to palm bends away from it (hyperextension of proximal inter-phalangeal joint).

2. Boutonniere deformity: In this deformity the joint in finger nearest to palm gets bent towards the palm (flexion of proximal inter-phalangeal joint) and the joint nearest to finger tip bends back (hyperextension of distal interphalangeal joint).

3. Ulnar deviation: In this swelling in the metacarpophalangeal joints (joint that connect bone in palm to the bone in finger ). It leads to leaning of the fingers away from thumb towards little fingers. This deformity is also known as ulnar drift.

\section{Manifestations in body parts other than joints}

- Eyes: Here it can lead to cataract (clouding of clear lens), dry eyes and scleritis (inflammation / redness in the white part of eye)

- Lungs: Inflammation, scar tissue, fibrosis can arise in lungs

- Skin: Rheumatoid nodules can form here. These refer to hard bumps under the skin. They are not painful and move easily on being touched. They mostly form especially on the elbows. They can also form in lungs and heart in later stages.

- Kidneys: Here it may lead to amyloidosis (disease arising from build up of an abnormal protein called amyloid in any tissue or organ).

- Heart: It can lead to inflammation of lining of heart (pericarditis), inflammation of heart muscle (myocarditis) and increase risk of cardiovascular disease (narrowing or blockage of blood vessels supplying heart that can lead to a chest

- Nerve tissue: Here it can cause peripheral neuropathy (damage to the nerves outside of the brain and spinal cord that causes weakness, numbness and pain, mainly in hands and feet) and carpal tunnel syndrome (pain, numbness, tingling in fingers from compression of the median nerve).

- Blood vessels: These may get inflamed and there may be formation of atheromatous plaques (accumulation of hard material in inner layer of artery wall) which includes lipids and calcium. It tends to narrow artery and hinders in blood flow

- Blood: It may lead to anaemia here

- liver: Is very rarely affected

- it can weakens immune system and make a person prone to infections

- on muscles it can lead to muscle weakness

- it can affect mucous membrane and lead to Sjogren's Syndrome (syndrome mainly characterised by dry eyes and dry mouth).

\section{What Causes Rheumatoid Arthritis and what are the risk factors}

The exact cause behind rheumatoid arthritis is not clearly understood. However it is thought to be an autoimmune disease.

What triggers the autoimmune process for RA is not exactly known. But there are certain factors that increase the risk of the disease. First among them is genetic factor. Certain genes (human leucocyte antigen HLA - DR1 and HLA DR4) make a person more prone to develop this disease. Females are more at risk as compared to males. Persons of middle age group are more affected. Other factors that put a person at risk of this include a family history of this disease, smoking, obesity, environmental exposures like exposures to asbestos or silica, stress, history of viral infections like Epstein - Barr virus (EBV) and certain dietary factors.

\section{Investigations for Rheumatoid Arthritis}

The blood test done in cases suspected of rheumatoid arthritis are rheumatoid factor, Anti - cyclic citrullinated peptide (anti - CCP), ESR and c - reactive protein (CRP). Other tests include X-Ray and MRI of affected joints which are done to rule out the severity of the disease.

\section{What are its types?}

Seropositive: In this type persons with rheumatoid arthritis have their blood tests positive for rheumatoid factor or anti - CCPs.

Seronegative: A person may develop Rheumatoid arthritis even in the absence of rheumatoid factor or anti-CCPs. This is termed as seronegative type rheumatoid arthritis. In this type the blood tests are negative for RF and antiCCPs. In such cases diagnosis is done on the basis of clinical symptoms and $\mathrm{X}$ - rays. This type is usually milder than the seropositve type.

Juvenile rheumatoid arthritis: Juvenile rheumatoid arthritis is when rheumatoid arthritis affects children younger than 17 yrs of age. It is also known as juvenile idiopathic arthritis (JIA).

Palindromic rheumatism: In this recurrent attacks of pain, 
swelling appears in one or more joints all of sudden. It last for few hours or few days. In between the flare up attacks the pain and swelling subsides completely. It is not a type of rheumatoid arthritis but people having palindromic rheumatism have chance to develop rheumatoid arthritis. Pathophysiology of Rheumatoid Arthritis

It is an autoimmune disease. Interplay between genetic susceptibility (people having certain genes human leucocyte antigen HLA - DR1 and HLA - DR4 are susceptible to this disease) and environmental factors (such as smoking) are thought to trigger the autoimmune process here.

In rheumatoid arthritis, the immune cells attacks the synovial lining of the synovial membranes surrounding the joints and cause its inflammation and thickening. It may further lead to destruction of cartilage and bone.

\section{Homeopathic treatment of Rheumatoid Arthritis}

Rheumatoid arthritis can be treated naturally with homeopathic medicines. These medicines helps to reduce inflammation of the joints by moderating the immune system. They also help to slow down further progression of the disease. The pain, swelling and stiffness in the joints are also relieved wonderfully with them. The joint damage and joint deformity though can't be cured but can be symptomatically managed with homeopathic medicines. These medicines are of natural origin thus are safe to use among people of all age groups. Commonly used medicines to treat it

\section{Rhus Tox - To relieve stiffness and pain in joints}

Rhus Tox helps to relieve joint pains and stiffness by reducing the joint inflammation. Persons needing it mainly have joint stiffness worse in the morning and also after a period of inactivity. Warm applications and motion of affected joint helps to relieve their symptoms. Massaging the joint also offer relief in them.

\section{Apis Mellifica - For reducing swelling in joints}

Apis Mellifica is a well indicated medicine for cases where joint is highly swollen. Along with this the joint is red, and inflamed. Joint pains which are mainly burning, stinging type are also well treated with Apis. Joint is also sensitive to touch.

\section{Bryonia - For red, hot, swollen joints}

Bryonia is helpful for cases where joints are red, hot and swollen. With this there is pain in joints. The pains are sharp, stitching type. The pain gets worse by motion and better with rest. Warmth may relieve the pains. It is Indicated when mostly knee and elbow joints are involved.

\section{Antimonium Crudum - To reduce pain in finger joints}

It is very beneficial to treat pain in finger joints and reduce its inflammation. Persons needing it mainly have worsening of pain from cold. They find relief by warm applications. Drawing sensation in the fingers may also be felt.

\section{Actaea Spicata - For wrist pain and swelling}

Actaea Spicata is mainly given for cases where wrist pain and swelling are present. Wrist is also red with heat. The pain is worse from motion. It is also indicated to treat pain and swelling in other small joints of fingers, toes and ankle.

\section{Caulophyllum - For small joints}

Caulophyllum is also used for treating arthritis that affect small joints. The small joints are painful and stiff in cases needing it. The joints of fingers, toes, ankle and wrist are affected in such cases. The pains keeps on shifting in minutes from one joint to other. In some case nodes are present on finger joints.

\section{Arnica - For markedly tender joints}

Arnica is majorly used when the joints are extremely tender. Person has great fear of touching the affceted joint due to pain and tenderness. The knee also has sore, bruised pain along with intense swelling.

\section{Stellaria Media - for pains in almost all the joints}

Stellaria Media is indicated for cases where for pains is felt in almost all the joints. The joints are sore to touch. Stiffness is also there in the joints. Sometimes the pain shifts from one joint to another.

\section{Ledum Pal - For painful, swollen ankles}

Ledum Pal offers great help in reducing pain and swelling in the ankles.

Persons needing it have worsening of ankle pain from movement. They find relief in it by cold applications. They may have ascending rheumatism where pain start from feet joints and then ascend to joints above it in the leg.

\section{Calcarea Carb For pain and swelling in knee joint}

It is an Important medicine to manage pain and swelling in the knee joint. The pains are drawing, tearing in nature. The knee is swollen mostly at night time. It is also hot and tenderness to touch.

\section{Colchicum - For pain that worsens from touch}

Persons having joint pain that worsens from touch are highly benefited with this medicine. Along with this they have heat and stiffness in the joints. The joints mainly involved where it is indicated are wrist, ankle, finger sand toes. Most times the pain are worse at night time in them. It is also used in management of cases where finger joints are highly damaged and distorted.

\section{Sanguinaria Can - for shoulder joint pain}

Sanguinaria Can works well to treat shoulder joint pain. It also helps to reduce attending stiffness in it. Shoulder pain gets worse by raising the arm in cases needing it. Worsening if pain at night is also noted in such cases.

\section{Pulsatilla - For shifting pain}

Pulsatilla is highly recommended medicine to treat shifting pains in joints. . The pain keeps on shifting. The affected joints are swollen and red. It is also indicated for joint pain in wet weather.

\section{Causticum and Guaiacum - for managing cases with joint deformity}

Causticum and Guaiacum both are excellent medicines to manage advanced cases having joint deformity. Though these medicines can't reverse the joint damage and deformity. But they can help to reduce pain and stiffness in joints. They also help to manage swelling in the affected joints. 
Some other rare medicines to treat cases of rheumatoid arthritis

Apart from above commonly used medicine some other rare medicines to treat cases of rheumatoid arthritis include Lithium Carb, Ruta, Salicylic Acid, Calcarea Phos, Ferrum Met, Lactic acid and Natrum Sulph. The key symptoms to use these medicines are as follows.

a) Lithium Carb: Pain, swelling and tenderness in small joints. Relief in joint pain from hot applications. Node formation of joints.

b) Ruta: Stiffness and pain in feet and hand joints. Node formation on wrist joint.

c) Salicylic Acid: Joint pain worsen from touch and motion. Shifting pains in joints.

d) Calcarea Phos: For joint pain worsening in cold weather

\section{Discussion}

Rheumatoid arthritis is a chronic inflammatory disorder causing joint inflammation with pain, swelling and stiffness of joints. These symptoms usually begin from small joints of hands or feet and can later affect other large joints too. It tends to involve multiple joints at a time and in a symmetrical way. It is an autoimmune disorder which means that the body's own defence cells start to destroy own healthy tissues. In rheumatoid arthritis the immune cells cause inflammation leading to destruction of the joints. It primarily affects joints but can also affect other organs like eyes, skin, lungs, heart etc. If not treated well in time then it progresses and can cause joint deformity and lead to disability. This disease has a strong genetic basis and tends to run in families.

\section{Conclusion}

RA is a debilitating, chronic, inflammatory disease, capable of causing joint damage as well as long-term disability. Early diagnosis and intervention are essential for the prevention of serious damage and loss of essential bodily functions. The treating physician should consider

adhering to treat-to-target (T2T) recommendations, by first outlining the aims and then implementing the protocols to achieve and assess them. Furthermore, early referral to a specialist can help to ensure better treatment outcomes. With advances in the field of molecular medicine, we have a better understanding of disease mechanisms which can aid in the designing of more effective treatments. Old treatment modalities have been optimized and new ones have been produced.

\section{References}

1. Lee JE, Kim IJ, Cho MS, Lee J. A Case of Rheumatoid Vasculitis Involving Hepatic Artery in Early Rheumatoid Arthritis. J Korean Med Sci 2017;32(7):1207-10.

2. Fox CQ, Ahmed SS. Physician Assistant's Clinical Review Cards. Philadelphia: F. A. Davis Company; 2002, 138-9.

3. McInnes IB, Schett $\mathrm{G}$. The pathogenesis of rheumatoid arthritis. N Engl J Med 2011;365(23):2205-19.

4. Chaudhari K, Rizvi S, Syed BA. Rheumatoid arthritis: current and future trends. Nat Rev Drug Discov 2016;15(5):305-6.

5. Picerno V, Ferro F, Adinolfi A, Valentini E, Tani C, Alunno A. One year in review: the pathogenesis of rheumatoid arthritis. Clin Exp Rheumatol 2015;33(4):551-8.

6. Alamanos Y, Voulgari PV, Drosos AA. Incidence and prevalence of rheumatoid arthritis, based on the 1987 American College of Rheumatology criteria: a systematic review. Semin Arthritis Rheum 2006;36(3):182-8.

7. Chopra A, Abdel-Nasser A. Epidemiology of rheumatic musculoskeletal disorders in the developing world. Best Pract Res Clin Rheumatol 2008;22(4):583-604.

8. McGonagle D, Hermann KG, Tan AL. Differentiation between osteoarthritis and psoriatic arthritis: implications for pathogenesis and treatment in the biologic therapy era. Rheumatology (Oxford) 2015;54(1):29-38.

9. Piyarulli D, Koolaee RM. A 22-Year-Old Female with Joint Pain. In: Piyarulli D, Koolaee RM, editors. Medicine Morning Report: Beyond the Pearls. Philadelphia: Elsevier 2016 65-77.

10. Staheli LT. Lower extremity management. In: Staheli LT, Hall JG, Jaffe KM, Paholke DO, editors. Arthrogryposis: A Text Atlas. Cambridge: Cambridge University Press 1998, 55-73.

11. Smolen JS, Aletaha D, Barton A, Burmester GR, Emery P, Firestein GS et al. Rheumatoid arthritis. Nat Rev Dis Primers 2018;4:18001. 